\title{
Heterogeneous Electric Vehicle Charging Coordination: A Variable Charging Speed Approach
}

\author{
Konstantina Valogianni \\ IE Business School \\ konstantina.valogianni@ie.edu
}

\author{
Wolfgang Ketter \\ University of Cologne \\ ketter@wiso.uni-koeln.de
}

\author{
John Collins \\ University of Minnesota \\ jcollins@cs.umn.edu
}

\author{
Gediminas Adomavicius \\ University of Minnesota \\ gedas@umn.edu
}

\begin{abstract}
We present a coordination mechanism that reduces peak demand coming from EV charging, supports grid stability and environmental sustainability. The proposed mechanism accounts for individual commuting preferences, as well as desired states of charge by certain deadlines, which can serve as a proxy for range anxiety. It can shape EV charging toward a desired profile, without violating individual preferences. Our mechanism mitigates herding, which is typical in populations where all agents receive the same price signals and make similar charging decisions. Furthermore, it assumes no prior knowledge about EV customers and therefore learns preferences and reactions to prices dynamically. We show through simulations that our mechanism induces a less volatile demand and lower peaks compared to currently used benchmarks.
\end{abstract}

\section{Introduction \& Background}

Electric vehicles (EVs) are important contributors to a sustainable future. They are independent from oil prices and are more energy efficient [11], which makes them more environmentally-friendly since they have reduced carbon footprint. More importantly, if EVs are charged with renewable energy, they contribute to environmental sustainability even more: both by emitting less $\mathrm{CO}_{2}$ from their engines and by requiring carbon-free energy for their charging. Their ability to increase sustainability levels makes them very popular in societies that strive to reduce their negative impact on the environment.

With rising EV adoption, new challenges arise for sustainable societies. All these new EVs added to the electricity grid will put its infrastructure under critical strain, since extra demand will be added to the grid. This demand was previously covered by gasoline, but now the electricity grid operators need to be able to cover the demand coming from this new customer segment, namely EV owners. In order for the grid to be stable, reliable and able to service all EV customers, new infrastructure needs to be installed, able to accommodate this extra electricity demand [11]. However, this solution is unsustainable since more raw materials need to be consumed and the investment costs can be excessive: the European Network of Transmission System Operators for Electricity foresees $€ 150$ billion of investments in grid infrastructure in the coming 10 years [6].

To address this challenge, academic literature has proposed a variety of EV charging coordination mechanisms, that can reallocate the EV charging demand over time, so that electricity peaks are reduced and the capacity utilization of the infrastructure increases. These coordination mechanisms are clustered into two categories. On the one hand, top-down coordination mechanisms assume the presence of a central entity (such as a grid manager or a system operator) that can exogenously intervene to induce a desired demand profile in an area. This intervention might be the interruption of consumption in some areas so that the total grid remains balanced. These type of approaches offer the benefit of fast grid stabilization, since a third party intervenes. The main disadvantage of these methods is that they do not always respect customer preferences and might end up violating people's comfort (e.g. with interruption of consumption).

On the other hand, bottom-up charging coordination mechanisms assume no formal coordinator. These approaches communicate price signals to the EV customers and observe their reactions. These signals are most of the time designed to incentivize energy consumption during low demand periods and provide counter-incentives for consuming during high demand periods. A typical example is the night tariff schemes that are available in numerous EU and US areas. These approaches allow customers to decide based on their schedule without violating their preferences. An important disadvantage is, however, that since all 
customers receive the same price signals, they behave in a similar way. This means that they are inclined to consume electricity when prices are low, creating new peaks during these periods. This phenomenon is known as herding or avalanche effect [14]. In Table 1 we present a summary of EV charging coordination mechanisms, categorized as bottom-up and top-down.

Table 1. Top-down and bottom-up EV charging coordination literature summary.

\begin{tabular}{|c|c|c|c|}
\hline & Top-down & & Bottom-up \\
\hline [1]: & $\begin{array}{l}\text { Aggregated EV } \\
\text { capacity coordination }\end{array}$ & [2]: & $\begin{array}{l}\text { EV coordination for } \\
\text { voltage regulation }\end{array}$ \\
\hline [3]: & $\begin{array}{l}\text { Dual implementation for } \\
\text { EV charging shifting based } \\
\text { on a central auctioneer }\end{array}$ & [5]: & $\begin{array}{l}\text { Decentralized EV } \\
\text { coordination using } \\
\text { temporal and } \\
\text { locational flexibility }\end{array}$ \\
\hline [21]: & $\begin{array}{l}\text { Online auction-based EV } \\
\text { charging mechanism }\end{array}$ & [9]: & $\begin{array}{l}\text { (A)synchronous } \\
\text { EV charging }\end{array}$ \\
\hline [12]: & $\begin{array}{l}\text { A fleet operator manages } \\
\text { EV charging }\end{array}$ & [10]: & $\begin{array}{l}\text { Locally optimal EV } \\
\text { scheduling protocol }\end{array}$ \\
\hline [22]: & $\begin{array}{l}\text { Pre-commitment auctions } \\
\text { for EV charging coordination }\end{array}$ & [15]: & $\begin{array}{l}\text { EV coordination for } \\
\text { frequency regulation }\end{array}$ \\
\hline
\end{tabular}

In addition to all previous pure top-down or bottom-up approaches, there is a set of hybrid methods, which assume the presence of a coordinating entity combined with high degree of decentralization on the EV owner's side. [20, 19] present a general framework of decentralized EV charging in which the objectives of the coordinator and the individual EV owners are weighted accordingly, toward a valley-filling or a cost-minimizing objective. Furthermore, [8, 9] present a decentralized charging coordination protocol combined with the presence of EV aggregator (grid manager/utility). The objective of this protocol is to achieve valley-filling or to track a given profile, after observing customer reactions.

The last set of hybrid methods has the ability to overcome herding by combining bottom-up and top-down objectives, making them very powerful and easily applicable. One shortcoming of those approaches is the modeling limitations when it comes to EV owner heterogeneous preferences. For example, EV owners, besides requiring sufficient electricity to drive, they also desire a certain state of charge in their battery so that they feel they have enough electricity to cover unexpected or spontaneous driving. This desired state of charge could serve as a proxy for range anxiety [7], since it varies across EV owners and expresses the desired maximum amount of energy in the battery by a certain time. EV owners with high range anxiety will require a higher desired state of charge than others with lower range anxiety. This parameter is important to study because, as [16] conclude, there is a need for extra infrastructure to accommodate this anxiety without threatening grid stability.

To address the above challenges, we present a coordination mechanism based on [23], which combines benefits from both bottom-up and top-down approaches and prevents herding in EV charging, while at the same time satisfies the heterogeneous customer preferences (driving deadlines, desired state of charge, etc.). Unlike previous work (e.g., [23]) which assumes that EV customers decide on EV charging based only on minimizing their costs, we use multiple objectives, since in reality EV customers have two conflicting objectives: satisfy their desired state of charge objective and minimize charging costs. We show how heterogeneous preferences (such as different levels of desired state of charge) influence peak demand and volatility. We observe that the desired state of charge is a key determinant for peak demand since higher levels of this parameter result in higher peaks and consequently higher needs for grid expansion.

Our coordination mechanism uses price functions of charging rate, rather than simple price values. It assumes that the energy price each consumer faces, is a function of the charging rate (speed). Consequently, a more impatient consumer that wants an EV charged quickly, will select a higher charging rate and will pay a higher price per energy unit (e.g. price/kWh). A more patient consumer can select a lower charging rate and reduce her cost for EV charging. With this approach, a grid manager can create disincentives for high demand peaks in EV charging. Unlike prior work that assumes one charging rate available $[8,9]$, we integrate the charging rate variable in the price function, allowing EV owners to select the rate which satisfies their heterogeneous preferences. Furthermore, our mechanism is adaptive and can quickly adjust the price functions to the needs of the particular consumer portfolio. This is done purely by observing and learning consumer behaviors, without assuming any prior knowledge about individual customer preferences.

\section{Model Description}

We assume that each individual EV owner wants to minimize her EV charging cost and at the same time reach a desired state of charge by a certain deadline (e.g. by the end of the day so that she has enough energy to drive the next morning). These two goals are conflicting since reducing costs means reducing $\mathrm{EV}$ charging and satisfying the desired state of charge objective increasing EV charging to the desired battery level. Therefore, we model the problem as a multi-criteria decision making and we present the decision maker, EV owner, with the full solution spectrum. Afterwards, the EV owner can decide about the desired charging plan (solution). In this way, we account in real time 
for the human behavior and heterogeneous preferences for different charging plans. A schematic overview of the proposed mechanism is displayed in Figure 1 (the sequence of steps is numbered in parenthesis). The dashed lines in Figure 1 indicate multiple instances of intelligent agents, assisting EV owners.

First, (1) the grid manager broadcasts price functions of power $(\mathrm{kW})$. Second, (2) the EV agent representing the EV owner, retrieves arrival and departure preferences from its owner, so that it can schedule EV charging at the minimum cost. Then, (3) it receives as input the desired state of charge for this particular EV owner (e.g. up to $90 \%$ of the EV's nominal capacity). Having all required inputs, (4) the EV agent schedules the charging so that cost is minimum but also the state of charge satisfies the desired state of charge by a certain deadline. Finally, the agent presents the set of optimal solutions to the EV owner and (5) she selects the preferred one.

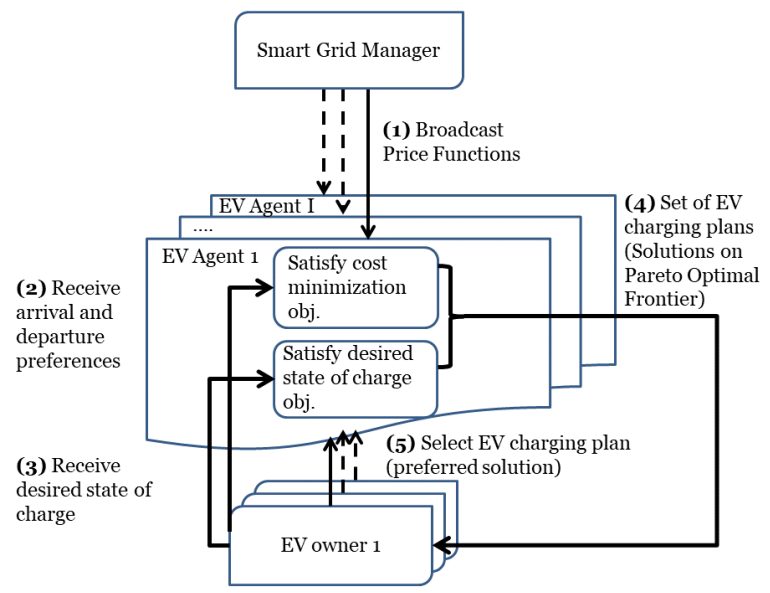

Figure 1. EV charging coordination overview.

As shown in Figure 1 the two decisions an EV agent has to make (minimize costs and satisfy the desired state of charge objective) are conflicting. Therefore, there are many solutions that satisfy this multi-criteria decision problem. These solutions constitute the Pareto optimal frontier of the problem and the EV owner herself decides on the preferred solution. To solve this multi-criteria decision problem we implement a multi-objective optimization problem which we solve with a genetic algorithm (GA) approach. Specifically, we implement an elitist GA [4] which selects the best solution for each point on the Pareto optimal frontier. This approach (unlike single objective problems) allows the decision maker (EV owner) to decide about the preferred solution choosing from the full solution space, rather than reducing it to one single optimal solution. Therefore, it allows for representing all types of heterogeneous preferences such as people that want higher state of charge and are willing to pay more or people that want to have reduced electricity costs and are willing to compromise their comfort by reducing their desired state of charge.

Modeling the heterogeneity of EV owners does not allow for single objective optimization and is better solved by metaheuristics such as GAs. However, GAs do not provide guarantees for convergence. NSGA-II [4], the variant of GAs we are deploying to solve this problem, is generally performing very well in providing the optimal solution spectrum and in all our simulation scenarios converged fast, but still it does not have convergence guarantees. Therefore, in the future, we plan examine the trade-off between richness of heterogeneous preferences and guarantees for convergence.

\subsection{Modeling Assumptions}

Below we describe the assumptions that draw the boundaries of our coordination mechanism.

1. EV owners are represented by self-interested intelligent agents that do not exchange information among each other. They only interact with the grid.

2. The interaction of the EV agents with the grid is limited to receiving price signals (retail prices) and decide on EV charging speed and duration, based on these prices. The prices are communicated to the EV agents in advance of their planning horizon.

3. EV owners have preferences regarding departure/arrival times and desired state of charge, that are unknown to the grid. The grid only observes their charging demand in reaction to prices.

4. All EVs are located and driving under the same distribution network, to avoid charging in other distribution networks.

5. The granularity of our analysis is 1 hour. This parameter is configurable and can be adjusted based on the problem's needs to e.g., 1-min or 15-min, etc.

6. The grid manager is in control of steering the aggregate EV charging consumption towards a desired profile which might be either a less volatile demand curve or a demand curve that follows the production pattern of a renewable production unit (e.g. wind turbine).

\subsection{EV's Decision Problem}

EV customers are represented by intelligent agents $i \in \mathbf{I}$ that are self-interested (they represent their owners preferences) and wish to minimize energy cost over 
a time horizon $\mathbf{T}=\{1 \ldots T\}$, while at the same time maximize the EV battery's state of charge to the desirable level. This level is different among individuals and can depend on their range anxiety [7]. The time horizon $\mathbf{T}$ is discretized to time intervals $t \in\{1 \ldots T\}$ of equal or non-equal duration.

The energy cost over $\mathbf{T}$ for each EV customer is $i$ the sum of costs for each interval:

$$
\sum_{t=1}^{T} c_{t}^{i}=\sum_{t=1}^{T} e_{t}^{i} \cdot P_{t}(\cdot)
$$

where $c_{t}$ is the cost of energy during time $t, e_{t}^{i}$ is the energy consumed in $\mathrm{kWh}$ during the interval, and $P_{t}(\cdot)$ is the (possibly rate-dependent) price of energy during this time. If we assume time intervals of duration $\Delta t \neq 0$ and charging at a constant rate $r_{t}^{i}$ in $\mathrm{kWh}$, then $e_{t}^{i}=r_{t}^{i} \cdot \Delta t$.

Each $\mathrm{EV}$ agent $i$ has a set of preferences $\boldsymbol{\theta}_{\boldsymbol{i}}$, including arrival $t_{a, i}^{n}$ and departure times $t_{d, i}^{n}$ over the horizon $T$ : $\boldsymbol{\theta}_{\boldsymbol{i}}=\left\{t_{a, i}^{n}, t_{d, i}^{n}\right\} \forall n \in \mathbf{N}^{\mathbf{i}}$, where $\mathbf{N}^{\mathbf{i}}$ is the set of intervals during which the vehicle $i$ is connected to a charger based on the user's driving profile. The timing of each interval (i.e. $\left\{t_{a, i}^{n}, t_{d, i}^{n}\right\}$, as well as the cardinality of the set $\left(N^{i}\right)$ are different across individuals $i$. The EV customer's decisions over a time horizon $\mathbf{T}$ are described by the following double objective:

$$
\begin{cases}\min _{\left[r_{1}^{i *} \ldots r_{T}^{i *}\right]} \sum_{n=1}^{N^{i}} \sum_{t=t_{a, i}^{n}}^{t_{d, i}^{n}} r_{t}^{i} \cdot \Delta t \cdot P_{t}\left(r_{t}^{i}\right) & : \text { Objective 1 } \\ \max _{\left[r_{1}^{i *} \ldots r_{T}^{i *}\right]} \sum_{n=1}^{N^{i}} \sum_{t=t_{a, i}^{n}}^{t_{d, i}^{n}} r_{t}^{i} \cdot \Delta t & : \text { Objective 2 }\end{cases}
$$

subject to (3)-(5).

Constraint (3) ensures that the total amount charged in the EV battery by a certain deadline $(T)$ is maximized up to reaching each agent's $i$ desired state of charge, $\operatorname{SoC}_{d}^{i}$. This desired state of charge is not a hard constraint, instead it represents an upper bound of electricity the customers are willing to purchase so that they can cover unexpected or spontaneous driving. The hard constraint which ensures a minimum electricity in the battery so that the driving needs are covered is described by (5).

$$
\sum_{n=1}^{N^{i}} \sum_{t=t_{a, i}^{n}}^{t_{d, i}^{n}} r_{t}^{i} \cdot \Delta t \leq S o C_{d}^{i}
$$

Constraint (4) sets the bounds for charging rate per timeslot $t$ (maximum charging speed):

$$
0 \leq r_{t}^{i} \leq r_{\max } \quad \forall t \in \mathbf{T}
$$

where $r_{\max }$ is the highest allowable rate offered by the charging infrastructure and it may vary per locations. The vector $\left[r_{1}^{i *} \ldots r_{T}^{i *}\right]$ has zero elements when $t \notin \boldsymbol{\theta}_{\boldsymbol{i}}$.

Each EV agent spends energy $E_{t}^{i}$ during the times $t$ the EV is driving. These timeslots can be $\forall t \notin \boldsymbol{\theta}_{\boldsymbol{i}}$ but not necessarily, because the car might be unplugged but also not driving. The state of charge of the battery for every timeslot $t$ is $S o C_{t}^{i}=S o C_{t-1}^{i}+r_{t}^{i} \cdot \Delta t-E_{t}^{i}$, which is equal to the state of charge in the timeslot $t-1, S o C_{t-1}^{i}$, and the amount charged during timeslot $t, r_{t}^{i} \cdot \Delta t$, (if the $\mathrm{EV}$ is available for charging) reduced by the amount of energy spent for driving during this timeslot, $E_{t}^{i}$, (if the $\mathrm{EV}$ is driving). Therefore, the amount charged at every timeslot $t$ should be:

$$
r_{t}^{i} \cdot \Delta t \geq S o C_{t}^{i}-\left(S o C_{t-1}^{i}-E_{t}^{i}\right) \quad \forall t \in \mathbf{T}
$$

Constraint (5) ensures that an EV agent charges at least an energy $E_{t}$ per timeslot $t$ that the EV is available for charging. The minimum amount required to charge at every timeslot $t$ should be $r_{t}^{i} \cdot \Delta t=S o C_{t}^{i}-\left(S o C_{t-1}^{i}-\right.$ $\left.E_{t}^{i}\right) \forall t \in \mathbf{T}$ so that the estimated driving needs of the EV owner are covered. We assume the $S o C^{i}$ is at a starting value $S o C_{0}^{i}$ at the beginning of horizon $T$.

Based on the presented multi-objective optimization problem the EV agent decides on a set of charging rate vectors in the form $\mathbf{r}^{\mathbf{i} *}=\left[r_{1}^{i *} \ldots r_{T}^{i *}\right]$. When the $\mathrm{EV}$ is not available for charging $\forall t \notin \boldsymbol{\theta}_{\boldsymbol{i}}$, the respective $r_{t}^{i *}$ are zero.

The problem described by equations (2)-(5) has more than one optimal solutions. These solutions - in absence of any further information - cannot be said to be better than the other. All these solutions constitute the efficient Pareto frontier of the problem and the decision maker has to select the preferred solution. To solve this multi-objective optimization problem we deploy a genetic algorithm (GA), and specifically, we implement the Non-Dominated Sorting Genetic Algorithm-II (NSGA-II) [4], which can provide the full spectrum of optimal solutions with low computational complexity and fast convergence.

\subsection{Smart Grid Manager's Decision Problem}

The grid manager steers the EV customer demand toward a desired profile. Therefore, we propose a pricing mechanism which is a function of charging rate and provides disincentives for EV customers to charge at high speeds when it is not urgent. This pricing scheme (Equation (6)) varies across time, and redistributes parts of the peak demand so that herding is prevented.

$$
P_{t}\left(r_{t}\right)=P_{0, t}+\alpha_{t} \cdot r_{t}
$$


The variable $r_{t}$ is the charging rate (power consumption) during timestep $t$ and $P_{0, t}$ is the price for zero demand and can either be constant, or be exogenously determined as e.g. a) the wholesale price of electricity at time $t$ or b) another variable price that is known ahead of time. The grid manager's goal is to determine $\alpha_{t}$ at each timestep $t$ that will produce the desired aggregate demand profile. The coefficient $\alpha_{t}$ determines the slope of the price curve with respect to the charging rate.

The coefficient $\alpha_{t}$ is the steering parameter of the pricing mechanism, since by increasing $\alpha_{t}$ the smart grid manager can increase the prices of the various charging speeds, increasing the prices of energy, as well. With this mechanism we introduce time as an extra dimension in the EV customers' decision: customers can reduce their electricity costs by reducing the charging speed, instead of reducing their actual energy consumption (which is the case with the traditional pricing schemes). Furthermore, by adjusting $\alpha_{t}$ dynamically, our pricing mechanism achieves peak demand redistribution without creating new peaks.

Since the grid manager is in control of steering the aggregate demand $\left(\mathbf{D}=\left[D_{1} \ldots D_{T}\right]\right)$, she sets prices so that the summation of all individual demand profiles for every time $t$ comes as close as possible to the desired demand $\left(D_{t} \approx \sum_{i=1}^{I} r_{t}^{i *}\right)$. The EV drivers' preferences are unknown to the grid manager and therefore it is unlikely to achieve an exact match of the desired aggregate demand and the summation of individual demands (i.e $\left.D_{t}=\sum_{i=1}^{I} r_{t}^{i *}\right)$. Thus, next we present an adaptive component whereby the grid manager observes the outcome of its actions on the EV driver population and adjusts future actions accordingly.

2.3.1 Adaptive Component Since grid managers have no prior knowledge about the heterogeneous EV owner preferences, they observe reactions to prices and adjust the parameters $\alpha_{t}$. We introduce an adaptive component in the coordination algorithm that makes it converge to the desired profile, $\mathbf{D}=\left[D_{1} \ldots D_{T}\right]$, without having knowledge about the EV agent population. This makes our mechanism flexible, since potential additions of agents with different preferences or drop-outs of existing agents can be observed online and the mechanism can adapt its behavior.

We build our adaptive component based on Iterative Learning Control (ILC) theory [18], modified to account for multiplicative adjustment factors, since this expedites convergence to the desired demand profile. Our algorithm acts in real-time and, therefore, it is crucial to react quickly and adapt on time to changes in the consumer portfolio. Specifically, the smart grid manager observes and stores the deviations of the observed demand and the intended profile. Based on these observations it updates the error function over horizons T, $\sum_{t=1}^{T} \varepsilon_{t}=\sum_{t=1}^{T} D_{t}-\sum_{t=1}^{T} \sum_{i=1}^{I} r_{i, t}^{*}$ and adjusts the value of $\alpha$ for the next period T based on an agent's learning factor $\lambda \in[1, M]$ (where $M$ sufficiently large number) so that the observed demand approximates the intended demand profile. The learning factor $\lambda$ varies across grid managers and we experiment with different values in our simulation. The adaptive component updates the next value of $\alpha_{t+T}$ based on the following rule:

$$
\alpha_{t+T}=\left\{\begin{array}{cl}
\lambda \cdot \alpha_{t} & : \sum_{t=1}^{T} \varepsilon_{t}<\sum_{t=1}^{T} \varepsilon_{t, \text { min }} \\
\frac{1}{\lambda} \cdot \alpha_{t} & : \sum_{t=1}^{T} \varepsilon_{t}>\sum_{t=1}^{T} \varepsilon_{t, \text { min }}
\end{array}\right.
$$

This decision rule is repeated by the control agent until the error term $\sum_{t=1}^{T} \varepsilon_{t}$ gets reduced to the desired error level $\sum_{t=1}^{T} \varepsilon_{t, \text { min }}$.

\section{Evaluation}

To evaluate our mechanism's performance we build a simulation environment based on Power TAC [13]. Power TAC is a smart grid simulation platform, where energy brokers compete to attract electricity customers and make profits. We display scenarios with various heterogeneous EV customer preferences and price function attributes (Table 2).

Table 2. Simulation Scenarios. Scenario Attributes

$$
\begin{gathered}
\alpha_{t}=\text { constant }, P_{0, t}=R_{t}\left\{\begin{array}{l}
S o C_{d}^{i}=50 \% \text { nom. cap. } \\
S o C_{d}^{i}=75 \% \text { nom. cap. } \\
S o C_{d}^{i}=100 \% \text { nom. cap. }
\end{array}\right. \\
\alpha_{t}=\text { variable, } P_{0, t}=R_{t}\left\{\begin{array}{l}
S o C_{d}^{i}=50 \% \text { nom. cap. } \\
S o C_{d}^{i}=75 \% \text { nom. cap. } \\
S o C_{d}^{i}=100 \% \text { nom. cap. }
\end{array}\right.
\end{gathered}
$$

First, we present results with the coefficient $\alpha_{t}=$ constant in the price function. We run this scenario in $\mathrm{EV}$ populations with $50 \%, 75 \%$ and $100 \%$ of the nominal battery capacity as the desired state of charge $\left(S_{o} C_{d}^{i}\right)$. The $50 \%$ is selected as the lowest scenario, because with a state of charge below $50 \%$ the driving needs of EV owners might not be covered. Second, we present results with $\alpha_{t}=$ variable in populations with $50 \%, 75 \%$ and $100 \%$ of the nominal battery capacity as the desired state of charge $\left(\operatorname{SoC}_{d}^{i}\right)$. The constant factor of price function (6), $P_{0, t}$, is calibrated with the corresponding energy price in each hour $\left(R_{t}\right)$, representing the electricity generation costs, taxes and network fees. 


\subsection{Simulation \& Data Description}

Below we describe the data we use to build our simulation environment.

3.1.1 Individual Preferences We calibrate our simulation with arrival and departure preferences obtained by the Bureau of Statistics in an EU country. This data includes different population clusters (full-time employees, part-time employees, students, retired persons, etc.) with a variaty of habits and driving behaviors (business commuting, leisure time driving, vacation, visits to relatives, shopping etc.). For each individual we get a driving profile with certain activities and driving demand for each activity, combined with arrival and departure times.

3.1.2 Electricity Prices To calibrate the $P_{0, t}$ factor of function (6), we use as an example of wholesale prices offered by the European Power Exchange (EPEX) adjusted to account for network fees, taxes and VAT for the EU country in question. In Figure 2 we show 3 weeks of retail price data, as they are used in the simulation.

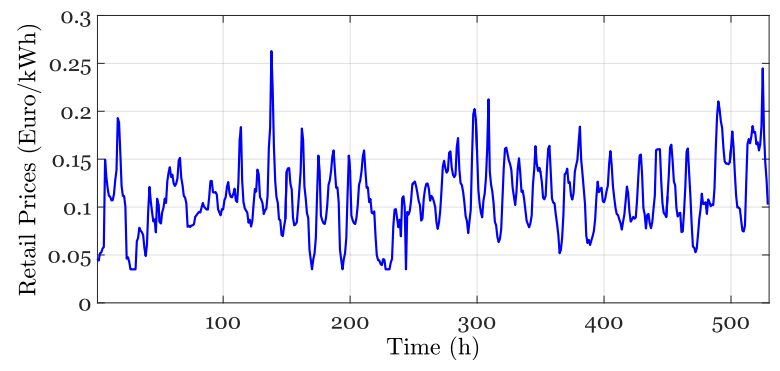

Figure 2. Retail prices (€/kWh) over 3 weeks.

3.1.3 Household Demand without EVs Finally, we want to evaluate the effect of EV charging on top of each EV customer's household demand. To calibrate this parameter we use data from households in the EU country in question obtained in collaboration with a European energy utility. In Figure 3 we show exemplary individual power demand curves (anonymized for privacy reasons). We observe that there are differences across households in the peak demand and the time intervals that this peak demand appears.

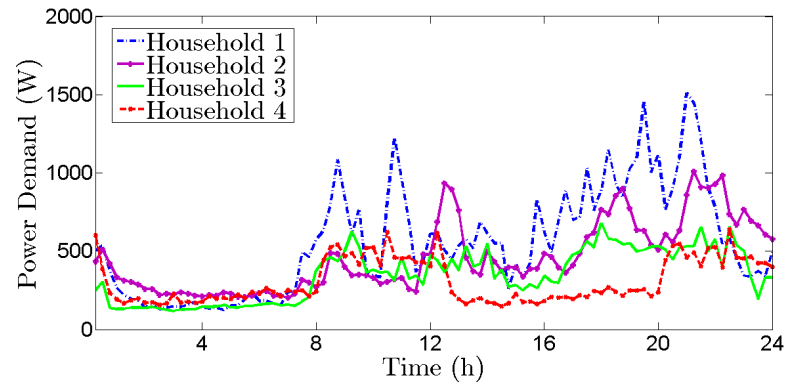

Figure 3. Examples of household power demand of some customers in the population on a random day.

\subsection{Numerical Example}

Next, we demonstrate how our mechanism works on a randomly selected EV customer. We assume an EV customer who owns a Nissan Leaf and her EV battery has storage of $24 \mathrm{kWh}$. This user indicates to the agent (handling the EV charging) that she ideally would like her battery up to $100 \%$ charged per day. The intelligent agent, after getting this input together with her arrival/departure preferences (that can be inserted directly or learned from past behavior), schedules EV charging using equation (2), subject to constraints (3)-(5). For this example we also assume that the grid manager broadcasts price functions with $\alpha_{t}=$ constant, $\forall t \in \mathbf{T}$. The parameters of this scenario are: $\alpha_{t}=$ constant, $P_{0, t}=R_{t}, S o C_{d}^{i}=100 \%$ of nominal capacity (24kWh).

The intelligent agent, after accounting for the preferences the EV owner provided as inputs, presents the following EV charging solution spectrum to the user (Figure 4). The y axis in Figure 4 is intentionally inversed so that it shows that a higher state of charge will incur higher costs. The GA converged after creating 187 generations of solutions and the Pareto frontier consists of 77 points (77 possible solutions). All these candidate solutions together with the EV customer's household demand profile (Figure 3) give the demand curve in Figure 5. The error bars outline the spectrum of solutions the EV customer can choose from.

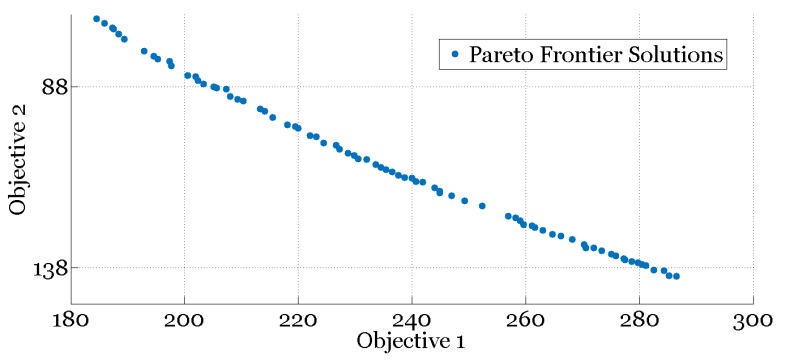

Figure 4. Pareto frontier with optimal EV charging plans (y-axis inversed). 


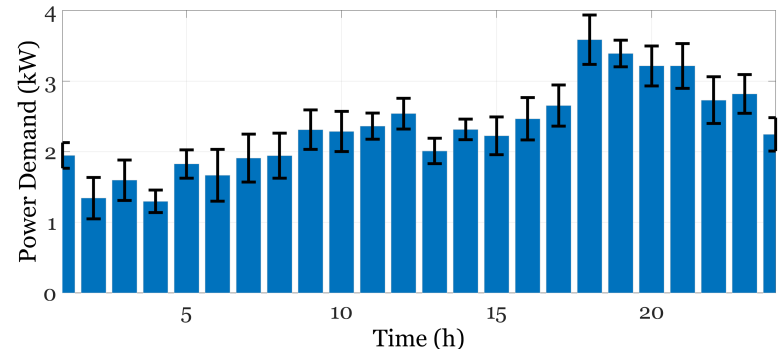

Figure 5. Example demand profile (household and EV demand).

\subsection{Impact of $S o C_{d}^{i}$ on Energy Demand}

In this section we examine how different levels of desired state of charge, $S o C_{d}^{i}$ influence the demand curve and consequently the need for extra infrastructure on the smart grid. We display results from the scenarios described before. The $S o C_{d}^{i}$ numbers are just used as examples and can be substituted with others without loss of generality of our results. We first start with assuming $\alpha_{t}=$ constant and we show that even constant $\alpha_{t}$ achieves redistribution of charging over time. Next, we show how adjusting $\alpha_{t}$ over time based on observed demand shapes the demand toward a desired profile.

3.3.1 Desired state of charge up to $S o C_{d}^{i}=50 \%$ of nominal capacity In this simulation scenario, the GA converged after creating 147 generations of solutions and generated the Pareto frontier consisting of 70 points (70 possible solutions). The steady state curve of all these solutions combined with the household demand of these customers is presented on the first graph of Figure 7. We observe in this graph that the error bars are either small or non-existent. This is a person who desires a charging up to $50 \%$ per day. This amount will just satisfy her driving needs leaving little energy for unexpected/spontaneous driving. Therefore, for this population segment the boundaries of Equations (3) and (5) are almost identical, yielding a narrow solution spectrum. This narrow solution spectrum can be also observed on the y-axis of Figure 6. The general intuition we get from the Pareto frontier solutions for this customer segment is that these customers do not charge any redundant amounts of energy since they only want to have just enough battery to drive. Also, for this type of customers both objectives have the lowest values because they charge the least amount of energy and, therefore, they have the lowest costs. This explains why this solution spectrum is located on the upper left part of Figure 6.

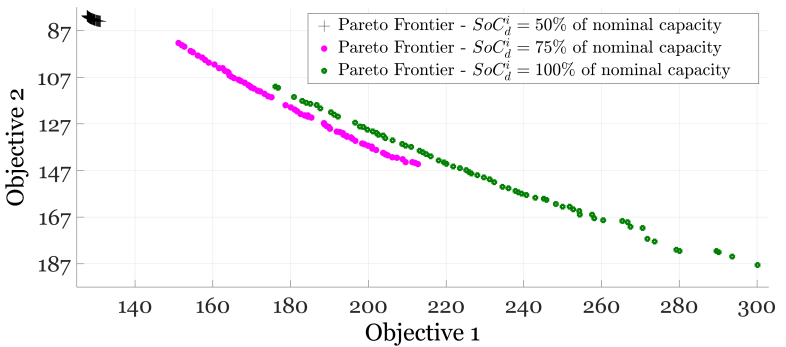

Figure 6. Pareto frontier with optimal EV charging plans, for customer segments with $S_{o} C_{d}^{i}=50 \%$, $S_{o} C_{d}^{i}=75 \%$ and $S o C_{d}^{i}=100 \%$ of nominal capacity (y-axis inversed).

3.3.2 Desired state of charge up to $S o C_{d}^{i}=75 \%$ of nominal capacity In this simulation scenario, the GA converged after creating 228 generations of solutions and generated the Pareto frontier consisting of 70 points (70 possible solutions). In this population segment, we observe that the spectrum of possible solutions is broader (error bars second graph in Figure 7). In this graph, we see that the error bars create higher peak demand compared to a population of customers with $S o C_{d}^{i}=50 \%$ of nominal capacity (first graph in Figure 7) during peak hours 18:00-21:00. In Figure 6, we observe that the solutions of this scenario are located in the middle of the graph. As expected, customers with a desired state of charge up to $S o C_{d}^{i}=75 \%$ of their nominal capacity charge more than customers with a desired state of charge up to $S o C_{d}^{i}=50 \%$ of their nominal capacity (higher values for Objective 2) and consequently, will pay more for energy (higher values for Objective 1).

3.3.3 Desired state of charge up to $S o C_{d}^{i}=100 \%$ of nominal capacity In this simulation scenario, the GA converged after creating 110 generations of solutions and generated the Pareto frontier consisting of 70 points (70 possible solutions). Here, we observe the highest peak demand of all three cases, since this EV customer segment desires their EV batteries charged up to full capacity at the end of each day (last graph Figure 7). Consequently, the EV agents increase the charging rates to achieve this goal within a day. Looking at Figure 6 we see that the solution spectrum for this customer segment is the broadest of all three customer segments. This happens because they require up to $100 \%$ battery charged and the agent has more options for charging (and creates a broader solution spectrum). Furthermore, we observe that some solutions overlap with some solutions from the previous customer segment. This happens again, because the $75 \%$ and the 
$100 \%$ of battery charged have an overlap in the spectrum of [lowest state of charge $-75 \%$ of nominal capacity]. Because of the broadest solution spectrum in this scenario, in the bottom graph of Figure 7 we observe the highest volatility, as well.
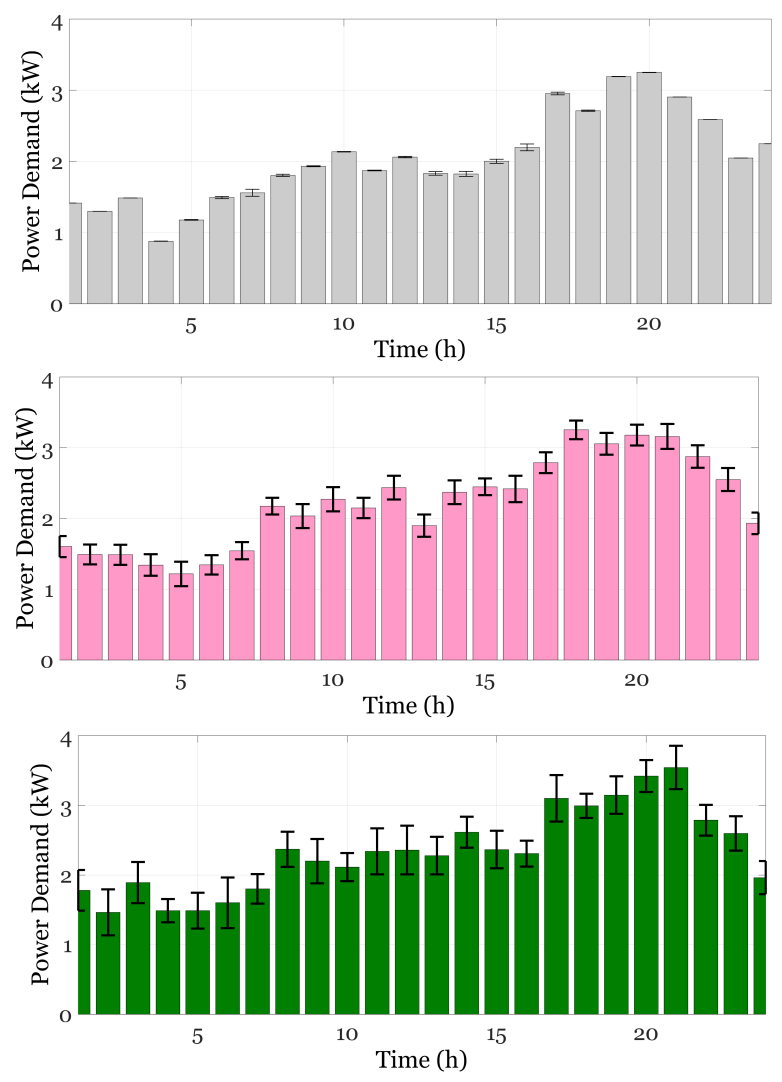

Figure 7. Household and EV demand, for customer segments with $S_{o} C_{d}^{i}=50 \%, S_{o} C_{d}^{i}=75 \%$ and $\operatorname{SoC}_{d}^{i}=100 \%$ of nominal capacity.

\subsection{Energy demand reshaping under variable price slope $\alpha_{t}$}

Next, we show how adjusting $\alpha_{t}$ over time creates different incentives and EV customers modify their charging accordingly. For the variable $\alpha_{t}$ scenarios we do not display the Pareto frontier for each iteration due to space limitations. After each period, though, a Pareto frontier is calculated and each EV customer selects one solution. We assume that EV customers have consistent preferences and each time they select the same type of solution (e.g., each time the same customer prefers the solution with the lowest cost or the highest battery capacity). In Figure 8 we display the EV charging demand disentangled from the household. We must note that after 31 iterations the changes in the EV charging demand are very small or non existent due to the individual arrival and departure preferences which are immutable constraints, together with the desired state of charge preferences. Also, the problem includes a lot of stochasticity (arrival and departure times, GA solution generation, etc.) and this leads to some volatility in the outcome after 31 iterations.

In Figure 8 we display the steady state curve of each customer population segment $\left(\operatorname{SoC}_{d}^{i}=50 \%\right.$, $S o C_{d}^{i}=75 \%$ and $S_{o} C_{d}^{i}=100 \%$ of nominal capacity). We see that in all three cases, after 31 iterations (31 times of adjusting the price slope $\alpha_{t}$ ), part of the charging demand is scheduled during the time interval [00:00-00:06] when the household demand is low and therefore, the EV and household combination yields an overall flatter curve. Also, we observe that after 31 iterations, the absolute peaks are reduced compared to iteration 1 (peak reduction for $S o C_{d}^{i}=$ 50\%: $14.90 \%$, SoC $_{d}^{i}=75 \%: 12.00 \%$, SoC $_{d}^{i}=100 \%$ : $8.01 \%$ ). We observe that increasing $S o C_{d}^{i}$ brings lower peak reduction, which is expected since with higher So $C_{d}^{i}$ the customers tend to charge more and at higher charging rates. However, the overall peak reduction shows that by adjusting the price slope $\alpha_{t}$ and learning from the EV customers' behavior, the grid manager can steer the demand towards a less volatile curve. There is still volatility in the demand that cannot be eliminated because of the immutable heterogeneous customer preferences (such as arrival and departures and desired state of charge). In future work, we will examine how to make the induced demand entirely flat, influencing the immutable customer preferences.

\subsection{Benchmarks}

To measure our mechanism's overall performance, we compare the peak demand and demand volatility induced by our mechanism and currently used benchmarks. To measure the demand volatility we use the peak-to-average ratio (PAR) [17]: $\left(P A R=\frac{r_{\text {peak }}}{r_{r m s}}=\right.$ $\frac{r_{\text {peak }}}{\sqrt{\frac{1}{T} \sum_{t=1}^{T} r_{t}^{2}}}$, which is also known as crest factor and measures the intensity of peaks or valleys in a curve. The results of this comparison are shown in Figure 9 and quantified in Table 3. The benchmarks we are using are listed below.

Benchmark 1: Real-world charging - Flat Pricing This benchmark accounts for EV charging during the period January 2013 - December 2013. This data set includes charging observations from 1500 charging poles in the whole EU country, from which the rest of our data comes. It has recordings of 10,462 EV owners and includes in total 231,976 transactions with the grid operator. All these transactions are completed under a 

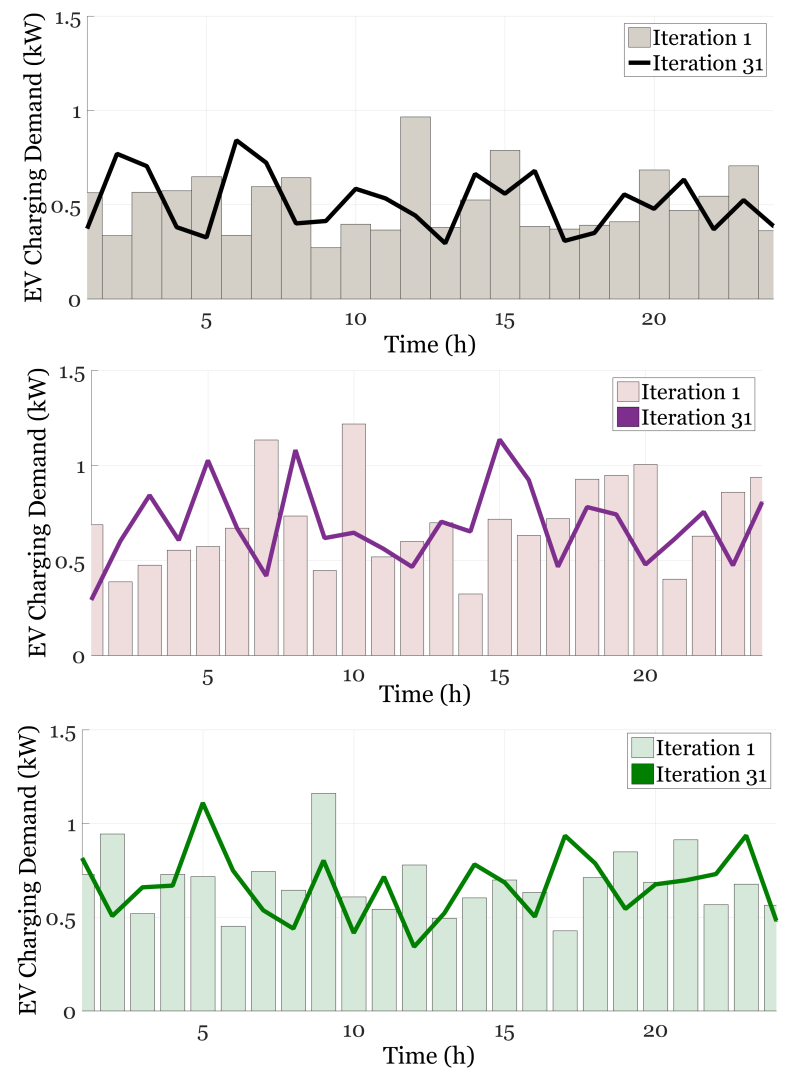

Figure 8. EV charging demand reshaping with variable $\alpha_{t}$, for customer segments with $\operatorname{SoC}_{d}^{i}=50 \%$, $S_{o} C_{d}^{i}=75 \%$ and $S o C_{d}^{i}=100 \%$ of nominal capacity.

flat pricing regime, which means that EV drivers were paying the same price per electricity unit, irrespective of the time of the day.

Benchmark 2: Rate-independent scenario - Variable Pricing This benchmark assumes self-interested agents, minimizing their costs based on a given variable retail price signal, which does not depend on the charging rate $\left(\alpha_{t}=0, \forall t \in \mathbf{T}\right)$, similarly to the flat pricing benchmark. However, in the variable pricing benchmark the prices of electricity vary across time intervals, i.e. $P=P_{0, t}$ non-constant over time.

These two benchmarks are representative of two different situations. The first one represents the current regime where no intelligent software is in place and therefore, the consumers charge based on convenience without price incentives. In our data we observed that in the EU country in question EV owners mostly charge during the day at their employers premises. This happens because modern companies incentivize their employees to adopt EVs by offering different benefits. Hence, the Benchmark 1 in Figure 9 has a peak that starts in the morning and continues till the evening.
The second benchmark represents the other extreme of the spectrum where all EV owners have intelligent software in place and decide on charging based on minimized costs. However, in this situation (dashed line in Figure 9) all EV owners receive the same prices and are incentivized to shift their EV charging in similar ways. Thus, we observe a significant peak during the night (00:00 till 05:00) which indicates all people shifted their EV charging during that time. This is an example of herding in charging. For this simulation experiment we assumed a heterogeneous EV owner population with all types of preferences represented.

Figure 9 and Table 3 show that our algorithm reduces both peak demand and PAR compared to the currently used benchmarks. Especially compared to the variable pricing benchmark, our mechanism reduces peak demand by almost $20 \%$ and volatility by $10 \%$. We also observe that the variable pricing benchmark creates higher peaks than the current EV charging under flat pricing (negative values in Table 3 ).

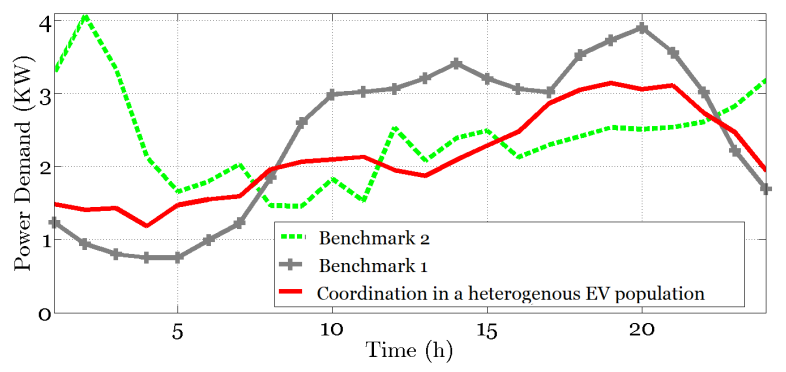

Figure 9. Benchmarking of the proposed coordination mechanism (household and EV demand).

Table 3. Energy PAR and Peak Reduction (negative values indicate increase).

\begin{tabular}{lrr}
\hline & $\begin{array}{r}\text { PAR red. } \\
(\%)\end{array}$ & $\begin{array}{r}\text { Peak red. } \\
(\%)\end{array}$ \\
\hline Coordination vs. Benchmark 1 & 3.50 & 15.85 \\
Coordination vs. Benchmark 2 & 10.31 & 19.31 \\
Benchmark 2 vs. Benchmark 1 & -7.60 & -4.29 \\
\hline
\end{tabular}

\section{Conclusions \& Future Work}

We presented a coordination mechanism that reduces peak demand coming from EV charging, supports grid stability and environmental sustainability. The proposed mechanism accounts for individual commuting preferences as well as desired state of charge, which can serve as a proxy for range anxiety. It can shape EV charging toward a desired profile, without violating individual preferences, while it mitigates herding. Furthermore, it assumes no prior knowledge about EV customers and, therefore, learns preferences 
and reactions to prices dynamically. We observed that increasing desired state of charge, and potentially increasing range anxiety on the EV customer's side creates higher demand volatility. Therefore, our mechanism can induce a smoother demand profile by observing and dynamically learning these attributes. In addition, we showed through simulations that our mechanism induces a less volatile demand and lower peaks compared to currently used benchmarks.

In the future, we plan to examine the trade-off between heterogeneous preferences and guarantees for convergence. Furthermore, currently EV chargers do not support continuous power intervals, therefore, to increase the practical applicability of our mechanism, we plan to examine the discrete optimization case of the presented continuous space solution.

\section{References}

[1] S. Acha, T. Green, and N. Shah. Optimal charging strategies of electric vehicles in the UK power market. In 2011 IEEE PES, Innovative Smart Grid Technologies (ISGT), pages $1-8$, January 2011.

[2] O. Beaude, Y. He, and M. Hennebel. Introducing decentralized ev charging coordination for the voltage regulation. In IEEE PES ISGT Europe 2013, pages 1-5. IEEE, 2013.

[3] K. De Craemer, S. Vandael, B. Claessens, and G. Deconinck. An event-driven dual coordination mechanism for demand side management of phevs. IEEE Transactions on Smart Grid, 5(2):751-760, March 2014.

[4] K. Deb, S. Agrawal, A. Pratap, and T. Meyarivan. A fast elitist non-dominated sorting genetic algorithm for multi-objective optimization: Nsga-ii. Lecture notes in computer science, 1917:849-858, 2000.

[5] R. D'hulst, F. De Ridder, B. Claessens, L. Knapen, and D. Janssens. Decentralized coordinated charging of electric vehicles considering locational and temporal flexibility. International Transactions on Electrical Energy Systems, 25(10):2562-2575, 2015.

[6] European Network of Transmission System Operators for Electricity. Ten-year Network Development Plan. ENTSOE, Brussels, Belgium, 2016.

[7] T. Franke, I. Neumann, F. Bühler, P. Cocron, and J. Krems. Experiencing range in an electric vehicle: Understanding psychological barriers. Applied Psychology, 61(3):368-391, 2011.

[8] L. Gan, U. Topcu, and S. H. Low. Stochastic distributed protocol for electric vehicle charging with discrete charging rate. In Power and Energy Society General Meeting, 2012 IEEE, pages 1-8. IEEE, 2012.

[9] L. Gan, U. Topcu, and S. H. Low. Optimal decentralized protocol for electric vehicle charging. IEEE Transactions on Power Systems, 28(2):940-951, 2013.

[10] Y. He, B. Venkatesh, and L. Guan. Optimal scheduling for charging and discharging of electric vehicles. IEEE Transactions on Smart Grid, 3(3):1095-1105, 2012.
[11] International Energy Agency. Global EV Outlook. Organisation for Economic Co-operation and Development, Paris, 2017.

[12] M. T. Kahlen, W. Ketter, and J. van Dalen. Electric vehicle virtual power plant dilemma: Grid balancing versus customer mobility. Production and Operations Management, pages 1-17, 2018.

[13] W. Ketter, M. Peters, J. Collins, and A. Gupta. Competitive benchmarking: An IS research approach to address wicked problems with big data and analytics. Management Information Systems Quarterly, 40(4):1057-1080, 2016.

[14] J.-H. Kim and A. Shcherbakova. Common failures of demand response. Energy, 36(2):873 - 880, 2011.

[15] H. Liu, Z. Hu, Y. Song, and J. Lin. Decentralized vehicle-to-grid control for primary frequency regulation considering charging demands. IEEE Transactions on Power Systems, 28(3):3480-3489, 2013.

[16] J. Neubauer and E. Wood. The impact of range anxiety and home, workplace, and public charging infrastructure on simulated battery electric vehicle lifetime utility. Journal of Power Sources, 257:12-20, 2014.

[17] H. K. Nguyen, J. B. Song, and Z. Han. Demand side management to reduce peak-to-average ratio using game theory in smart grid. In 2012 IEEE Conference on Computer Communications Workshops (INFOCOM WKSHPS), Orlando, FL, 2012.

[18] D. H. Owens and J. Hätönen. Iterative learning controlan optimization paradigm. Annual Reviews in Control, 29(1):57-70, 2005.

[19] J. Rivera, C. Goebel, and H.-A. Jacobsen. Distributed convex optimization for electric vehicle aggregators. IEEE Transactions on Smart Grid, 8(4):1852-1863, 2017.

[20] J. Rivera, P. Wolfrum, S. Hirche, C. Goebel, and H.-A. Jacobsen. Alternating direction method of multipliers for decentralized electric vehicle charging control. In Decision and Control (CDC), 2013 IEEE 52nd Annual Conference on, pages 6960-6965. IEEE, 2013.

[21] V. Robu, E. H. Gerding, S. Stein, D. C. Parkes, A. Rogers, and N. R. Jennings. An online mechanism for multi-unit demand and its application to plug-in hybrid electric vehicle charging. Journal of Artificial Intelligence Research, 48:175-230, 2013.

[22] S. Stein, E. H. Gerding, A. Nedea, A. Rosenfeld, and N. R. Jennings. Market interfaces for electric vehicle charging. Journal of Artificial Intelligence Research, 59:175-227, 2017.

[23] K. Valogianni, W. Ketter, and J. Collins. A multiagent approach to variable-rate electric vehicle charging coordination. Proceedings of the 14th International Conference on Autonomous Agents and Multi-Agent Systems (AAMAS15), pages 1131-1139, May 2015. 